\title{
TRATAMENTO ENDODÔNTICO DE PRÉ-MOLAR SUPERIOR COM TRÊS CANAIS: UM RELATO DE CASO
}

Root canal treatment of three-rooted maxillary premolar: a case report

Micheli Nádia Boneti ${ }^{1}$; Rafael Caranhato²; Bruno Emmanuelli³; Pauline Mastella Lang³ Simone Tuchtenhagen ${ }^{3}$; Caroline Pietroski Grando ${ }^{3}$

${ }^{1}$ Acadêmica de Odontologia da Universidade Regional Integrada do Alto Uruguai e das Missões - URI Erechim.E-mail: miicheli.boneti@gmail.com

${ }^{2}$ Acadêmico de Odontologia da Universidade Regional Integrada do Alto Uruguai e das Missões - URI Erechim.

${ }^{3}$ Professor(a) do Curso de Odontologia da Universidade Regional Integrada do Alto Uruguai e das Missões - URI Erechim.

Data do recebimento: 29/08/2019 - Data do aceite: 19/02/2020

RESUMO: A endodontia é a especialidade dentro da odontologia que se ocupa da prevenção, do diagnóstico e do tratamento de doenças ou lesões da polpa dental. Portanto, é imprescindível o conhecimento anatômico de cada elemento. É importante estar preparado para lidar com possíveis alterações anatômicas, já que $7,5 \%$ de pré-molares podem possuir três canais, número que vem crescendo ao longo dos anos. $\mathrm{O}$ objetivo deste estudo é relatar o caso de um tratamento endodôntico de um primeiro pré-molar superior com três canais radiculares, descrever a variação anatômica encontrada no primeiro pré-molar superior e discutir como se deve realizar o diagnóstico e o tratamento proposto para casos de primeiros pré-molares com três canais. $\mathrm{O}$ relato de caso clínico foi devidamente autorizado pelo paciente, e aprovado pelo Comitê de Ética em Pesquisa. Conclui-se que, para não haver a persistência de infecção no sistema de canais radiculares, são necessários a correta identificação e o tratamento. Assim, aumentam-se os índices de sucesso nos tratamentos endodônticos.

Palavras-chave: Endodontia. Dente Pré-Molar. Tratamento do Canal Radicular. 


\begin{abstract}
Endodontics is the specialty in dentistry that deals with the prevention, diagnosis and treatment of diseases or injuries of the dental pulp. Therefore, the anatomical knowledge of each element is essential. It is important to be prepared to deal with possible anatomical changes, $7.5 \%$ of premolars can have three root canals, that number has been growing over the years. The aim of this study is to report the case of an endodontic treatment of an upper first premolar with three root canals, describe the anatomical variation found in the maxillary first premolar and discuss how to diagnose and treat three-canals first premolar cases. The clinical case report was authorized by the patient, which was approved by the Research Ethics Committee. The conclusion is that in order to avoid persistent infection in the root canal system, proper identification and treatment is necessary, thus increasing the success in endodontic treatments.
\end{abstract}

Keywords: Endodontics. Bicuspid. Root Canal Therapy.

\section{Introdução}

A endodontia é a especialidade dentro da odontologia que se ocupa da prevenção, do diagnóstico e do tratamento de doenças ou lesões da polpa dental. O tratamento do canal radicular é um meio seguro e eficaz de preservar dentes que, em outras circunstâncias, estariam perdidos (COHEN; HARGREAVES, 2011).

A efetividade do tratamento endodôntico se deve à limpeza dos canais seguindo um correto preparo químico-mecânico, podendo ser utilizados instrumentos manuais ou rotatórios, sendo indispensável o uso de soluções irrigadoras. Para que haja sucesso a longo prazo é preciso obter um adequado selamento e obturação do sistema de canais radiculares (PORTELA et al., 2011; SIQUEIRA JR et al. 2017).

Um minucioso conhecimento da anatomia interna e um entendimento do potencial de variações são essenciais para o sucesso da terapia endodôntica (TOMAZINHO et al., 2008).
As maiores dificuldades encontradas em endodontia geralmente são por variações na anatomia dos canais, mas mesmo em canais únicos é importante um amplo conhecimento das possíveis variações a serem encontradas. O elemento dental que apresenta maior número de canais do que seu grupo dental representa um desafio adicional, começando pela abertura diferenciada, acesso a todos os canais, localização, limpeza, até a modelagem do sistema de canais. (SOARES; LEONARDO, 2003; MARQUES et al., 2019).

É extremamente importante que o dentista clínico conheça a anatomia dos elementos a serem tratados. A falta de conhecimento resulta em diagnósticos errôneos, erro na localização dos canais, e a incorreta ou incompleta preparação e obturação (AGWAN; SHEIKH, 2016; PRAVEEN et al., 2015).

Quando um dente possui um número maior de canais do que o habitual, requer um correto diagnóstico, avaliação criteriosa da radiografia e ainda se deve observar variações morfológicas da anatomia pulpar, antes de 
iniciar o tratamento (FERREIRA; MORAES; BERNARDINELI, 2000).

Devido à importância de uma correta identificação e conhecimento dos passos a serem seguidos frente a variações anatômicas, é importante que casos que se distanciem do padrão sejam publicados. Casos como de um primeiro pré-molar trirradiculado pertencem a variações anatômicas pouco frequentes, e um olhar diferenciado é exigido do cirurgião-dentista no momento de resolução do caso, pois seu nível de dificuldade aumenta. Desta forma, o relato para a comunidade científica é importante para que sirva de base para consulta, e ainda para pesquisas futuras.

O objetivo deste relato é apresentar o caso de um tratamento endodôntico de primeiro pré-molar superior com três canais radiculares, realizado no Centro de Estágios e Práticas Profissionais da URI Erechim (URICEPP), em termos de diagnóstico, tratamento e prognóstico, descrever a variação anatômica encontrada e discutir como se deve realizar o diagnóstico e o tratamento proposto para casos de primeiros pré-molares superiores com três canais.

\section{Material e Métodos}

Esta pesquisa foi aprovada pelo Comitê de Ética em Pesquisa da Universidade Regional Integrada do Alto Uruguai e das Missões - URI Erechim, sob o número CAAE 12781019.6.0000.5351, parecer $\mathrm{n}^{\mathrm{o}}$ 3.448.579, atendendo aos princípios éticos da pesquisa envolvendo seres humanos, de acordo com a resolução $n^{\circ} 466 / 2012$. Os descritores utilizados para a busca de artigos foram: endodontia, pré-molares, três canais radiculares, endodontics, premolars, three root canals. Assim, os artigos selecionados foram os que obtiveram um adequado delineamento como base de dados.

\section{Relato de Caso}

O paciente M.G., 25 anos, sexo masculino, procurou atendimento na URICEPP, no departamento de Odontologia, onde foi triado e encaminhado para a disciplina de Endodontia III. Foi realizada a anamnese, buscando avaliar o paciente como um todo, a queixa do paciente foi de dor no elemento 24 (primeiro pré-molar superior esquerdo). Constatado que o paciente não possuía nenhum outro problema sistêmico e possuía a pressão arterial em níveis normais, iniciou-se $\mathrm{o}$ atendimento odontológico. Antes de iniciar os procedimentos, em todas as consultas foram realizadas antissepsia intra e extraoral no paciente, com clorexidina. A resposta do paciente para o teste de percussão vertical foi positiva, e negativa para palpação apical, o elemento em questão não apresentava mobilidade, não houve necessidade clínica para realização de teste ao frio, devido à exposição do sistema de canais radiculares. Por meio da radiografia inicial ortorradial (Figura 1), percebe-se a fratura coronária no elemento 24 e exposição do sistema de canais radiculares com diagnóstico inicial de necrose pulpar, tendo como tratamento proposto a necropulpectomia.

\section{Figura 1}

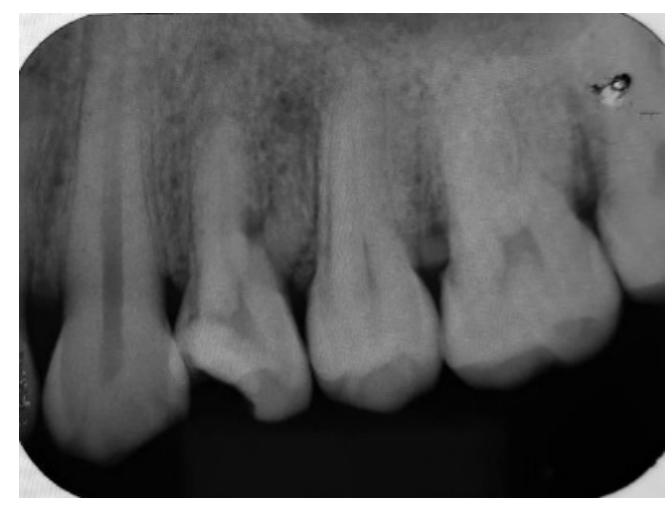


Realizada então anestesia com articaína 4\% (1: 100.000), para o nervo alveolar superior médio esquerdo, infiltrativa local palatina com complementação intraligamentar e intrapulpar durante os procedimentos, já que o paciente relatava dor durante o acesso e instrumentação. Foi constatada anatomia radiográfica diferenciada no elemento 24 e definido o CAD - Comprimento Aparente do Dente; com isso, após o isolamento absoluto e a abertura coronária, realizou-se inspeções na câmara pulpar a fim de localizar três canais radiculares (Figura 2), por meio da exploração dos canais radiculares com limas $\mathrm{K}$ série especial (Maillefer-Dentsply), \#8, \#10 e \#15 no CTEX - Comprimento de Trabalho de Exploração (comprimento aparente do dente $-3 \mathrm{~mm})$.

Figura 2

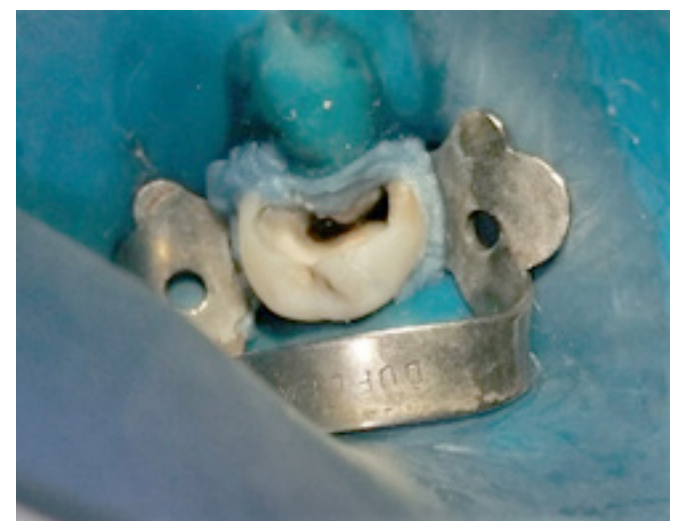

O preparo do terço cervical do canal radicular foi realizado com broca Gattes Glidden (Maillefer-Dentsply), \#3, \#2, \#1, respectivamente, a fim de remover o conteúdo necrótico, microrganismos, substratos e produtos bacterianos encontrados no interior dos canais radiculares; em cada troca de lima ou de brocas foi irrigado com hipoclorito de sódio na concentração de 2,5\% (Asfer Indústria Química), seguido de aspiração.

A odontometria (Figura 3) foi realizada segundo a técnica de Ingle, mensurando o dente a partir da radiografia inicial, realizada com posicionador odontológico esterilizável (Maquira Indústria de Produtos Odontológicos S.A), subtraído três milímetros, introduzida uma lima no interior de cada canal radicular e realizada nova radiografia. Com base nesta última radiografia, medida a distância entre a ponta do instrumento e o vértice radicular, e somada essa medida com o tamanho real do instrumento menos meio milímetro, que foi a margem de segurança estabelecida por Ingle para determinar o comprimento de trabalho. Definido o IAI (Instrumento Apical Inicial), este foi instrumentado no comprimento de trabalho (CT) de $18 \mathrm{~mm}$, para o canal palatino, $20 \mathrm{~mm}$ para o canal disto-vestibular e $21 \mathrm{~mm}$ para o canal mésio-vestibular.

Figura 3

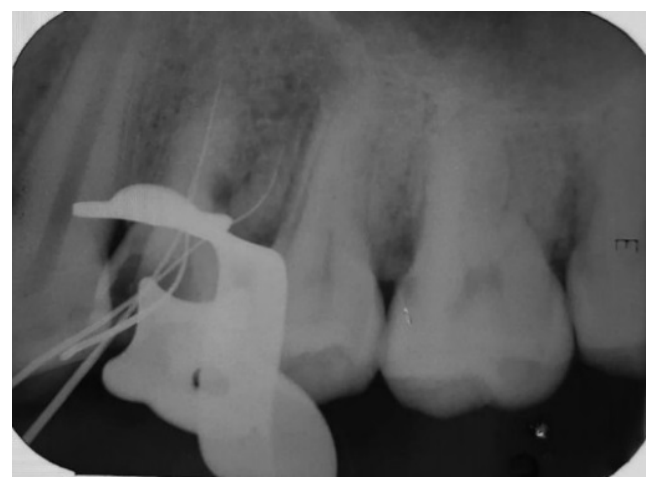

A instrumentação foi realizada manualmente, por meio da técnica Crown-Down, coroa-ápice, foi iniciada com lima calibre \#10 para os canais disto-vestibular e mésio-vestibular, e finalizados com lima calibre \#30; já o canal palatino teve sua instrumentação iniciada com lima \#25 e finalizado com lima \#45. Sempre, a cada troca de lima, realizada irrigação com hipoclorito de sódio 2,5\% (Asfer Indústria Química) e ainda realizada a patência foraminal utilizando lima calibre \#10 com 1mm a mais do que o comprimento de trabalho de cada canal para a prevenção 
de acúmulo de raspas de dentina e limpeza foraminal, que podem vir a comprometer o preparo do terço apical.

$\mathrm{O}$ recuo progressivo programado dos canais radiculares foi realizado com o objetivo de unir os terços dentinários. Realizado o toalete final com ácido diaminotetracético (EDTA) a 17\% líquido (Biodinâmica Química e Farmacêutica), agindo por meio da quelação, sequestrando íons de cálcio da dentina, formando quelatos de cálcio solúveis, fazendo com que haja maior penetração de agentes desinfetantes (SEN; WESSELINK; TURKUN; 1995), com agitação por meio de limas manuais durante 3 minutos, pois técnicas de ativação de irrigantes melhoram a remoção da smear layer e detritos presentes nos canais, comparando-se com a irrigação convencional somente com agulha (VIRDEE, et al., 2017); com isso, foi realizada então, a irrigação final com soro fisiológico com o objetivo de neutralizar o meio, seguido pela secagem dos canais radiculares e desinfecção dos cones de guta percha. Em seguida, a prova visual e tátil com cones de guta percha foi realizada, para obter-se uma confirmação radiográfica, por meio da conometria. Por fim, foi efetuada a obturação do sistema de canais radiculares por meio da técnica da condensação lateral e cimento endodôntico (AH Plus - Dentsply). Realizada radiografia de comprovação da qualidade da obturação (Figura 4), e complementada a condensação com o condensador de guta percha Mcspadden (Maillefer-Dentsply), seguida da condensação vertical e corte dos cones.

A restauração final provisória foi realizada com um selamento duplo de obturador provisório (Cavitec - Caithec Industrial) sob a embocadura dos canais e final com ionômero de vidro restaurador (Vidrion R S.S. White Duflex), foi checada a oclusão e então realização da radiografia final (Figura 5). Comprovando o correto selamento do sistema de canais radiculares. Em seguida, o paciente foi encaminhado para a realização da reabilitação protética com pino e prótese de cerâmica.

Figura 4

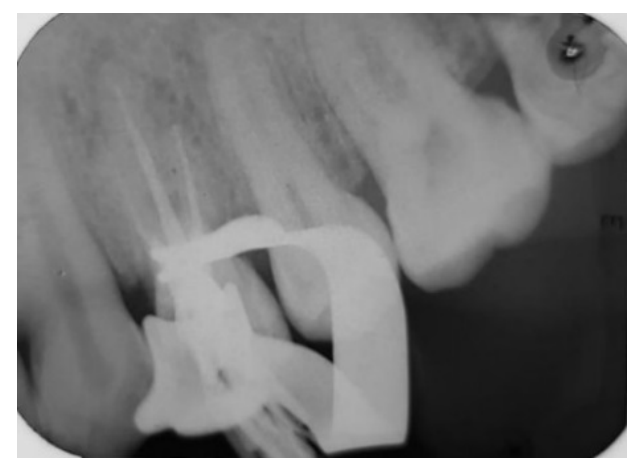

Figura 5

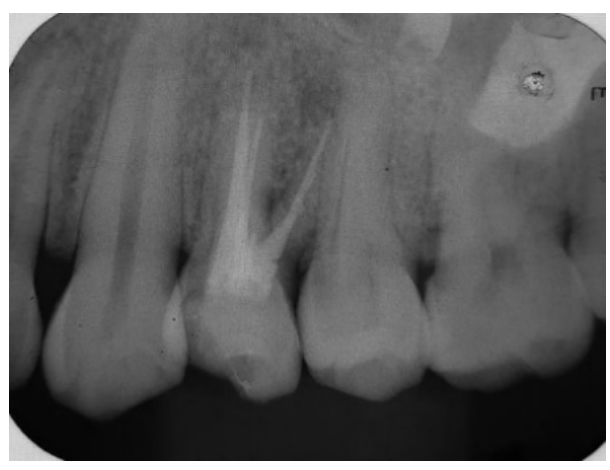

O tratamento endodôntico foi realizado em 2 consultas, com o tempo de espera de 7 dias. Como medicação intracanal foi realizada a inserção de pasta de hidróxido de cálcio em solução aquosa (UltraCal XS, Ultradent Products, Inc.). Sabe-se que o hidróxido de cálcio, com elevado nível de $\mathrm{pH}$, possui um efeito mineralizador e antibacteriano, devido a sua dissociação química em íons de cálcio e hidroxila, propiciando a inativação de enzimas bacterianas e ativando enzimas teciduais proporcionando também efeito mineralizador (ESTRELA et al., 1995). A restauração provisória interconsultas foi realizada com selamento duplo de obturador provisório 
(Cavitec - Caithec Industrial) e ionômero de vidro restaurador (Vidrion R - S.S. White).

\section{Discussão}

\section{Comparação deste caso com outros encontrados na literatura}

Ao comparar este caso clínico com outros casos, percebemos que a maioria dos autores não relataram o sal anestésico utilizado; por outro lado, Martins (2011) relatou também utilizar articaína. Li et al. (2018) conduziram um estudo para avaliar eficácia clínica e segurança da articaína quando usada para anestesia em casos de problemas pulpares, chegando à conclusão de que, quando comparada com a lidocaína, a articaína possui um melhor efeito analgésico e é segura para ser utilizada em paciente com problemas pulpares. Em umas revisões sistemáticas de literatura, Su et al. (2016); Nagendrababu et al. (2020) também concluem que tanto a articaína $4 \%$ como a lidocaína $2 \%$ são eficazes e seguras, porém a articaína $4 \%$ obteve um resultado superior para tratamentos de pulpite irreversível, possuindo início de ação mais rápido e ainda apresentando menor chance de reações adversas.

Quanto à solução irrigante, Tomazinho et al. (2008) e Uğur; Akpđnar; Altunbaş (2017) também fizeram o uso de hipoclorito de sódio na concentração de 2,5\% em seus casos clínicos; já Agwan; Sheikh (2016) e Soares; Leonardo (2003) utilizaram uma concentração menor de hipoclorito de sódio, na concentração de 1\%. No entanto, Martins (2011) utilizou uma concentração de hipoclorito de sódio de 5,25\%.

O hipoclorito de sódio é a solução irrigante mais utilizada, porém em termos de concentração, quantidade e tempo de utilização ainda não há consensos. Sendo assim, é importante considerar realizar trocas regulares e ainda utilizar uma quantidade regular para que a eficácia antibacteriana da solução de hipoclorito de sódio seja mantida, compensando assim a importância de sua concentração. (ARRUDA et al., 2019; SIQUEIRA et al., 2000).

\section{Variação anatômica do primeiro pré- molar superior}

Os primeiros pré-molares superiores possuem uma variação no número de raízes, número de canais, variação na configuração da câmara pulpar, as depressões presentes nas raízes limitam também a visualização do limite apical em radiografias (AGWAN; SHEIKH, 2016).

O primeiro pré-molar está situado na arcada dentária entre o canino e o segundo pré-molar. Possui duas raízes cônicas com inclinação apical distal, sendo a vestibular maior e lingual menor, em alguns casos essas raízes podem estar fusionadas, geralmente apresentam somente uma linha demarcatória nítida. Sua câmara pulpar se apresenta irregularmente cúbica e achatada na direção mésio-distal, as entradas dos canais se encontram debaixo de cada cúspide, seus canais raramente são divergentes (MADEIRA, 2007; PORTELA et al., 2011).

Na maioria dos casos, 84\% apresentam dois canais mesmo possuindo uma só raiz, são canais estreitos, mas sem maiores curvaturas, portanto não elevam a dificuldade do tratamento endodôntico. Quando encontrados três canais, geralmente são dois vestibulares e um palatino, sendo assim canais mais delgados que elevam o grau de dificuldade do tratamento endodôntico (SOARES; GOLDBERG, 2011).

Em um estudo sobre pré-molares inferiores, em 1986, Trope, Elfenbei e Tronstad constataram um fato curioso relacionado aos pacientes de raça negra. Nestes pacientes a chance de obterem pré-molares com mais de um canal foi três vezes maior do que entre 
pacientes brancos. Segundo sua pesquisa, até quatro entre dez pacientes negros tinham ao menos um pré-molar inferior com dois ou mais canais.

Há relatos de 1925 por Hess, Zürcher e Dolamore, que encontraram uma frequência de 0,5 a $0,6 \%$ da ocorrência de três canais; em 1979, Vertucci e Gegauff relatam que 5\% dos elementos têm três canais em seu ápice. Em 1998, Kartal, Özçelik e Cimilli encontraram uma porcentagem total de $1,66 \%$ de elementos com 3 canais em seu ápice, com porcentagens menores que outros estudos da época. Em 2007, Madeira relata que até $7 \%$ dos casos podem possuir três canais. Em 2011, Soares e Goldberg chegaram à porcentagem de 7,5\% dos casos com três canais.

Com o avanço dos estudos cada vez mais estão sendo encontradas maiores variabilidades anatômicas, exigindo do cirurgião-dentista clínico uma maior atenção ao realizar tratamentos endodônticos desse grupo dental.

\section{Importância do exame clínico e de imagens}

Antes de se iniciar qualquer tipo de tratamento, inclusive o endodôntico, é se suma importância que sejam minunciosamente analisadas as imagens radiográficas pré-operatórias do elemento em questão. Ainda é importante lembrar que um exame radiográfico é uma representação bidimensional de uma estrutura tridimensional, muitas vezes ocultando detalhes importantes (MATHEW et al., 2015; KARTAL; ÖZÇELIK; CIMILLI; 1998).

Uma tomada radiográfica angulada complementar permite a dissociação dos condutos e, consequentemente, sua visualização radiográfica, em casos em que as raízes são fusionadas, caso as três raízes forem separadas, a imagem radiográfica é semelhante à de um primeiro molar superior. Devido a essa semelhança anatômica, pré-molares superio- res com molares muitas vezes são chamados de minimolares ou radiculous (MATHEW et al., 2015; MAIBAUM, 1989; GOON, 1993).

Em pré-molares com três raízes superiores há uma diretriz geral para sua identificação, se a largura do terço médio da raiz aparece igual ou maior que a largura mésio-distal da imagem da coroa, provavelmente o dente terá três raízes. Porém não servindo para elementos trirradiculados com, por exemplo, dois canais em sua raiz vestibular (SIERASKI, 1989).

Outra evidência radiográfica da presença de mais de um canal, na mesma raiz ou em outra raiz independente, é quando há uma perda de radiolucidez abrupta ao longo do canal (PRAVEEN et al., 2015).

É importante observar o assoalho da câmara pulpar durante a abertura, pois ele pode apresentar vestígios dos tipos de canais radiculares existentes. Se há somente um canal, ele está no centro da câmara pulpar e é relativamente fácil de ser encontrado. Se um orifício for encontrado longe do centro, há maiores possibilidades de haver outro no lado oposto. Quanto mais afastadas as entradas dos canais, maior a chance de estarem separados por todo seu comprimento (MARTINS, 2011; KARTAL; ÖZÇELIK; CIMILLI, 1998).

A abertura final deve possuir um acesso em forma de $\mathrm{T}$ ou triangular, para permitir uma melhor visualização e acesso aos canais (MAIBAUM, 1989).

Após a abertura e remoção completa do teto da câmara pulpar, a percepção tátil pelo cirurgião-dentista é fundamental, deve-se observar a coloração do assoalho da câmara pulpar, sondar com sonda endodôntica retas e limas de menor diâmetro. Limas de exploração que não chegam ao comprimento total do canal e que, ao serem retiradas, apresentam a extremidade da lima angulada podem indicar uma variação na trajetória do canal, podendo 
ser uma divisão em 2 canais ou apenas uma curvatura. A simples utilização de corantes como o azul de metileno ajuda a evidenciar anatomias diferenciadas (MARTINS, 2011; DE MOOR; CALBERSON, 2005).

\section{Importância do diagnóstico e tratamentos adequados na previsibilidade clínica}

Para que qualquer tratamento endodôntico tenha sucesso é importante que se remova todo o tecido pulpar inflamado ou necrótico dos canais (KARTAL; ÖZÇELIK; CIMILLI; 1998).

Casos em que uma anatomia diferenciada não é percebida, resultam em falhas, o conhecimento sobre anatomia, morfologia e ainda estar preparado para enfrentar qualquer dificuldade encontrada são essenciais para o sucesso. Caso contrário, pode-se resultar em erro de diagnóstico, erro na identificação e localização do sistema de canais radiculares, uma limpeza, preparação e obturação incompletas ou errôneas (AGWAN; SHEIKH, 2016).

Erros mais graves podem levar à perda do elemento dental, muitas vezes cometidos pela falta de conhecimento da anatomia dental, como perfurações, desvios da anatomia original do canal e iatrogenias diversas (PORTELA et al., 2011).

Quando se conhecem as possíveis complicações e como diagnosticá-las, as chances de insucesso diminuem. Para auxiliar no diagnóstico clínico e radiográfico pode-se lançar mão do uso da magnificação, com microscópios operatórios e ainda tomografias computadorizadas cone-beam (PORTELA et al., 2011).

A maior causa de insucesso no tratamento endodôntico depende basicamente da dificuldade em localizar e até mesmo obturar canais com anatomias variadas. A incidência de canais ou raízes que não foram encontradas durante o tratamento endodôntico representam $42 \%$ dos casos em que há necessidade de realização de retratamentos (HOEN; PINK, 2002).

\section{Considerações Finais}

O estudo acerca da anatomia interna dentária é de fundamental importância na área endodôntica, e deve vir aliado a um correto diagnóstico, alicerçado na anamnese, no exame clínico e em exames complementares.

No tratamento deste relato de caso foi seguida a técnica de instrumentação manual Crown-Down, por se acreditar na redução de chances do transporte apical do canal.

São importantes o correto diagnóstico e a correta inspeção clínica do assoalho da câmara pulpar, assim como a remoção de todo o teto de câmara. Uma correta análise radiográfica e métodos auxiliares de ampliação, complementados com uma boa iluminação, tornam o tratamento destes tipos de anatomias menos comuns em tratamentos previsíveis e com bom prognóstico. 


\section{REFERÊNCIAS}

AGWAN, A. S.; SHEIKH, Z. Identification and endodontic treatment of three canalled maxillary first premolar. Journal of Ayub Medical College Abbottabad, v. 03, n. 28, p. 627-629, 2016.

ARRUDA, J. A. A.; SCHUCH, L. F.; PEREIRA, A.; MONTEIRO, J. L. G. C.; MELO-JÚNIOR, P. M. R.; MESQUITA, R. A.; MORENO A.; CALLOU, G. Investigation of different sodium hypochlorite volumes, concentrations and times of irrigation in endodontic therapy: a systematic review. Archives Of Health Investigation, v. 8, n. 4, p.185-191, 2019.

COHEN S.; BURNS R. C. Caminhos da polpa. 2. ed. Rio de Janeiro: Guanabara-Koogan, 1982. COHEN, S.; HARGREAVES, K. M. (Coord.). Caminhos da polpa. 10. ed. Rio de Janeiro: Elsevier, 2011.

DE MOOR R.; CALBERSON F. Root canal treatment in mandubular second premolar with three root canals. Journal Of Endodontics, v. 31, p. 310-313, 2005.

ESTRELA C.; BAMMANN L.L.; SYDNEY G.B.; MOUAR J. Efeito antibacteriano de pastas de hidróxido de cálcio sobre bactérias aeróbias facultativas. Revista da Faculdade de Odontologia de Bauru, v. 3, p. 109-114, 1995.

FERREIRA, C.; DEMORAES, I.; BERNARDINELI, N. Three-Rooted Maxillary Second Premolar. Journal Of Endodontics, v. 26, n. 2, p. 105-106, fev. 2000.

FILHO, F. J. S. Endodontia passo a passo: evidências clínicas [recurso eletrônico]. São Paulo: Artes Médicas, 2015.

GOON, W. W. The "radiculous" maxillary premolar: recognition, diagnosis, and case report of surgical intervention. Northwest Dentistry Journal, v. 72, n. 02, p. 31-33, 1993.

HESS, W.; ZÜRCHER, E.; DOLAMORE, W. H. The anatomy of the root-canals of the teeth of the permanent dentition. New York, William Wood. 1925.

HOEN, M.; PINK, F. Contemporary Endodontic Retreatments: An Analysis based on Clinical Treatment Findings. Journal Of Endodontics, v. 28, n. 12, p. 834-836, 2002.

KARTAL, N.; ÖZÇELIK, B.; CIMILLI, H. Root canal morphology of maxillary premolars. Journal Of Endodontics, v. 24, n. 6, p. 417-419, 1998.

LI, J; WEI, W; YANG, W; LI, J; GAO, Y; QIAN, Y. Comparision of clinical efficacy and safety between articaine and lidocaine in the anaesthesia management of tooth pulp disease. Pakistan Journal of Pharmaceutical Sciences, v. 31, n. 5, p. 2303-2306, 2018.

MADEIRA, M. C.; CRUZ-RIZZOLO, R. J. Anatomia do dente. 5. ed., rev. amp. São Paulo: Sarvier, 2007.

MAIBAUM, W. W. Endodontic treatment of a "ridiculous" maxillary premolar: A case report. General Dentistry, v. 37, p. 340-341, 1989.

MARQUES, E. F.; SILVA, D. M. S.; FROTA, D. R.; BITENCOURT, L. Endodontic Treatment of the first Maxillary Molar with 7 Root Canals: Case Report. International Journal Of Advanced Engineering Research And Science, v. 6, n. 12, p. 534-539, 2019.

MARTINS, J. N. R. Primeiro pré-molar superior com três canais: diagnóstico e tratamento - quatro casos clínicos. Revista Portuguesa de Estomatología, Medicina Dentária e Cirugia Maxilofacial, v. 52, n. 1, p. 43-51, 2011. 
MATHEW, J.; DEVADATHAN, A.; SYRIAC, G.; SHAMINI, S. Root canal treatment of a maxillary first premolar with three roots. Journal Of Pharmacy And Bioallied Sciences, v. 7, n. 6, p. 746748, 2015.

NAGENDRABABU, V.; DUNCAN, H.; WHITWORTH, J.; NEKOOFAR, M.; PULIKKOTIL, S.; VEETTIL, S.; DUMMER, P. Is articaine more effective than lidocaine in patients with irreversible pulpitis? An umbrella review. International Endodontic Journal, v. 53, n. 2, p.200-213, 2020.

PORTELA, C. P.; FILHO, F. B.; TOMAZINHO, F. S. F.; CORRER, G. M.; MORO, A.; MORESCA, R. C. Estudo da Anatomia Interna dos Pré-Molares - Revisão de Literatura. Odonto, v. 19, n. 37, p. 63-72, 2011.

PRAVEEN, R.; THAKUR, S.; KIRTHIGA, M.; SHANKAR, S.; NAIR, V. S.; MANGHANI, P. The ridiculous premolars: Case reports of a maxillary and mandibular premolar with three canals. Journal Of Natural Science, Biology And Medicine, v. 6, n. 2, p.442-445, 2015.

SEN, B. H.; WESSELINK, P. R.; TÜRKÜN M. The smear layer: a phenomenon in root canal therapy. International Endodontic Journal, v. 32, n. 3, p. 141-148, 1995.

SIERASKI, S. M.; TAYLOR, G. N.; KOHN, R. A. Identification and endodontic management of three-canalled maxillary premolars. Journal Of Endodontics, v. 15, n. 1, p.29-32, 1989.

SIQUEIRA JR, J. F.; PÉREZ, A. R.; MARCELIANO-ALVES, M. F.; PROVENZANO, J. C.; MONTEROS, S. G.; PIRES, F. R.; VIEIRA, G. C. S.; RÔÇAS, I. N.; ALVES, F. R. F. What happens to unprepared root canal walls: a correlative analysis using micro-computed tomography and histology/scanning electron microscopy. International Endodontic Journal, v. 51, n. 5, p.501-508, 2017.

SIQUEIRA JR, J.; ROCAS, I.; FAVIERI, A.; LIMA, K. Chemomechanical Reduction of the Bacterial Population in the Root Canal after Instrumentation and Irrigation with 1\%, 2.5\%, and 5.25\% Sodium Hypochlorite. Journal of Endodontics, v. 26, n. 6, p. 331-334, 2000.

SOARES, I. J.; GOLDBERG, F. Endodontia: técnica e fundamentos. 2. ed. Porto Alegre: Artmed, 2011.

SOARES, J. A.; LEONARDO, R. T. Root canal treatment of three-rooted maxillary first and second premolars - a case report. International Endodontic Journal, v. 36, n. 10, p.705-710, 2003.

SU, N.; LI, C.; WANG, H.; SHEN, J.; LIU, W.; KOU, L. Efficacy and safety of articaine versus lidocaine for irreversible pulpitis treatment: A systematic review and meta-analysis of randomised controlled trials. Australian Endodontic Journal, v. 44, n. 1, p.4-15, 2016.

TOMAZINHO, F. S. F.; VALENÇA, P. C.; BINDO, T. Z.; FARINIUK, L. F.; FILHO, F. B.; SCAINI, F. Tratamento endodôntico de pré-molares superiores com três raízes e três canais. Revista SulBrasileira de Odontologia, v. 1, n. 5, p.63-67, 2008.

TROPE, M.; ELFENBEIN, L.; TRONSTAD, L. Mandibular premolars with more than one root canal in different race groups. Journal of Endodontics, v. 12, n. 8, p.343-345, 1986.

UğUR, Z.; AKPđNAR, K. E.; ALTUNBAş, D. Maxillary first premolars with three root canals: two case reports. Journal of Istanbul University Faculty of Dentistry, v. 51, n. 3, p. 50-54, 2017.

VERTUCCI, F. J.; GEGAUFF, A.J. Root canal morphology of the maxillary first premolar. Journal of the American Dental Association, v. 99, n. 2, p.194-198, 1979.

VIRDEE, S. S.; SEYMOUR, D. W.; FARNELL, D.; BHAMRA, G.; BHAKTA, S. Efficacy of irrigant activation techniques in removing intracanal smear layer and debris from mature permanent teeth: a systematic review and meta-analysis. International Endodontic Journal, v. 51, n. 6, p. 605621, 2017. 